\title{
The influence of the menstrual cycle on diameter and respiratory collapsibility of inferior vena cava in the population of young, healthy women - preliminary results
}

\author{
Maria Nowak ${ }^{\circledR}$, Julia Dyda², Helena Barczyńska², \\ Maciej Ołubowicz², Alicja Radtke ${ }^{2}$, Marianna Turek², \\ Radosław Stanisław Nowak ${ }^{3} \odot$, Marcin Gruchała ${ }^{1}$ \\ ${ }^{1}$ First Department of Cardiology, Medical University of Gdańsk, Gdańsk, Poland \\ ${ }^{2}$ Student Scientific Circle of Cardiology, 1st Clinic of Cardriology, Medical University of Gdańsk, Gdańsk, Poland \\ ${ }^{3}$ Department of Cardiology and Electrotherapy, Medical University of Gdańsk, Gdańsk, Poland
}

\begin{abstract}
Introduction: Echocardiographic assessment of interior vena cava (IVC) is a part of estimation of right atrial pressure. In young women values exceeding norm are observed. The aim of our study was an echocardiographic assessment of IVC dimension depending on the phase of menstrual cycle among young, healthy women. Materials and methods: Female students of Medical University of Gdańsk were enrolled to the study. Each volunteer underwent echocardiographic examination of IVC diameter (d-IVC) and respiratory decrease in dimension in two time points, depending on the phase of menstrual cycle: in the first days of menstruation (Phase $M$ ), in the second part of menstrual cycle (Phase L). Results: 31 patients completed the study. There was a significant difference between the $d-I V C$ in Phase $M$ and Phase $L(1.98 \pm 0.25 \mathrm{~cm}$ vs $1.86 \pm 0.3 \mathrm{~cm} ; p<0.05)$. We did not observe correlation in terms of the inspiratory collapsibility. In the Phase $M 77 \%$ patients achieved at least $50 \%$ decrease in dimension during inspiration comparing to $87 \%$ in Phase $L(p=0.89)$. 35\% patients had $d$-IVC exceeding reference values. Conclusions: In population of young women diameter of IVC exceeding reference values can be observed. IVC dimension dependents on the menstrual cycle.
\end{abstract}

Keywords: echocardiography • menstrual cycle • inferior vena cava

\section{Citation}

Nowak M, Dyda J, Barczyńska H, Ołubowicz M, Radtke A, Turek M, et al. The influence of the menstrual cycle on diameter and respiratory collapsibility of inferior vena cava in the population of young, healthy women - preliminary results. Eur J Transl Clin Med. 2019;2(1):53-55.

DOI: $10.31373 /$ ejtcm/103002

Corresponding author:

Radosław Stanisław Nowak, Department of Cardiology and Electrotherapy, Medical University of Gdańsk, Gdańsk, Poland

e-mail: nowyrad@gumed.edu.pl

No external funds.

Available online: www.ejtcm.gumed.edu.pl

Copyright $®$ Medical University of Gdańsk

This is Open Access article distributed under the terms of the Creative Commons Attribution-ShareAlike 4.0 International. 


\section{Introduction}

Assessment of the inferior vena cava (IVC) from the subcostal window is part of a routine echocardiographic examination. Measurement of the IVC dimension and collapsibility during inspiration is used to noninvasively estimate right atrial pressure [1]. According to the 2010 American Society of Echocardiography guidelines, IVC diameter $<2.1 \mathrm{~cm}$ and inspiratory collapse $>50 \%$ correspond with normal right atrial pressure of 0-5 $\mathrm{mm} \mathrm{Hg}$ [2]. Clinical practice shows that IVC values exceeding norms can be observed as an isolated abnormality in the population of young women.

The influence of female sex hormones on the cardiovascular system is well-acknowledged. Estrogen and progesterone affect cardiac performance and have an important role in atheroprotection. In addition, those hormones have direct impact on vessels through non-genomic mechanisms [3]. Female sex hormones have an effect on body fluid regulation, induce dilatation of the peripheral vessels and decrease venous flow. Furthermore, estrogen affects the synthesis of nitric oxide, while progesterone has diuretic activity. The concentration of female sex hormones depends on cyclic changes and present the lowest level of estrogene and progesterone during firsts days of menstrual cycle [45]. There is a lack of studies regarding the influence of those changes on IVC diameter measurement.

Therefore, the aim of our study was an echocardiographic assessment of IVC dimensions depending on the phase of menstrual cycle among young, healthy women.

\section{Materials and methods}

Female volunteer students of Medical University of Gdańsk aged 19 to 30 were enrolled into the study. The exclusion criteria included pregnancy, use of

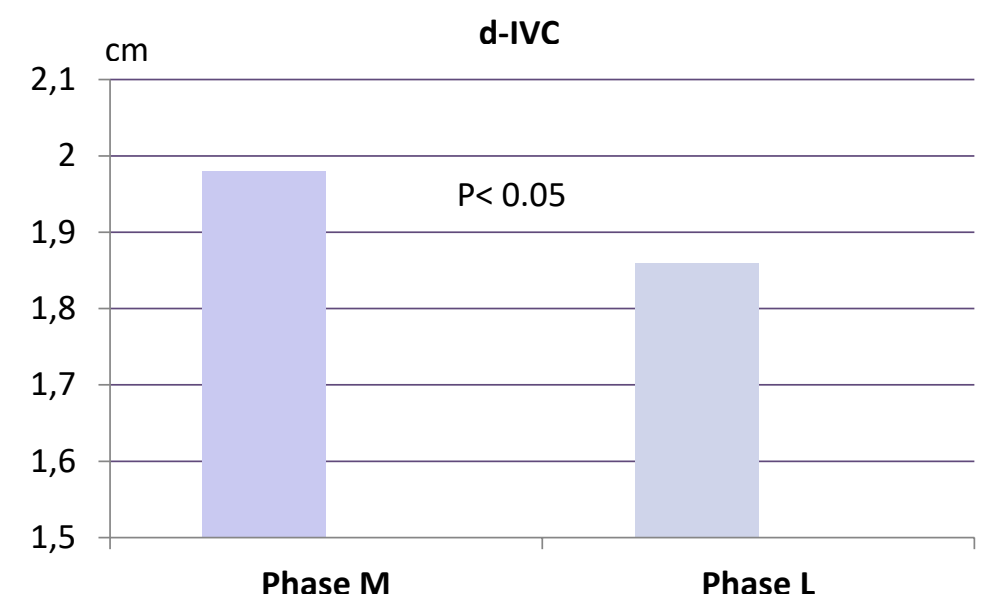

Figure 1. d-IVC depending on the phase of menstrual cycle hormonal contraception, menstrual disorders, cardiovascular and respiratory diseases and competitive sport activity. Each patient had 2 echocardiographic examinations of IVC diameter (d-IVC) and respiratory decrease in dimension in two time points: in the first days of menstruation (Phase $M$ ) and in the second part of cycle (Phase L). We estimated Phase $L$ as 14 days before beginning of the next cycle. All examinations were conducted on fasting volunteers. The d-IVC was measured while supine, in the subcostal window and in longitudinal projection, $3 \mathrm{~cm}$ from the right atrium outlet (Figure 1). IVC diameter $<2.1 \mathrm{~cm}$ was considered normal. Additionally, questionnaires concerning data about menstrual cycle characteristics (length, regularity, day of cycle during examination) fluid intake, amount of weekly physical exercise and anthropometrics were collected. Extended echocardiographic study was conducted in each volunteer with IVC abnormalities

\section{Statistical analysis}

All data were tabulated in MS Excel and analyzed using the standard Statistica v.12.0 package (StatSoft, Tulsa Inc., USA). Data with normal distribution (based on Shapiro- Wilk test) were compared with student's t-test. Non-normally distributed variables were compared with non-parametric test (Wilcoxson's test). Chi-squared test was used to analyze the number of cases between dichotomized subgroups. P-value $<0.05$ was considered statistically significant.

\section{Results}

31 volunteers completed the study. Average age was $23.7 \pm 4.2$ years and BMI $20.97 \pm 2.4 \mathrm{~kg} / \mathrm{m} 2$. There was a significant difference between the d-IVC in Phase $M$ and Phase $L(1.98 \pm 0.25 \mathrm{~cm}$ vs $1.86 \pm 0.3 \mathrm{~cm}$, respectively; $p$ $<0.05$; Figure 1). On the contrary, we did not observe that correlation in terms of the inspiratory collapsibility. In the Phase M 77\% patients achieved at least $50 \%$ decrease in dimension during inspiration comparing to $87 \%$ in Phase $L(p=0.89)$. Physical exercise time did not affect a d-IVC $(1.9 \pm 0.36 \mathrm{~cm}$ for $>2.5$ hours/week group and $1.87 \pm 0.23 \mathrm{~cm}$ for $\leq 2.5$ hours/week group; $p=0.74)$. Also, there was no difference in d-IVC due to the amount of fluid intake $(1.9 \pm 0.42 \mathrm{~cm}$ for $\geq 2$ liter/day group versus 
$1.88 \pm 0.2 \mathrm{~cm}$ for $<2$ liter/day group; $\mathrm{p}=0.8) .35 \%$ patients had $d$-IVC exceeding reference values at least in one examination but full echocardiography did not reveal any other clinically significant abnormalities.

\section{Discussion}

As the availability of the right heart catheterization is limited, echocardiographic methods to assess right atrial pressure including IVC measurement are commonly performed. In several causes (e.g. athletic training, obesity or narrowing of the IVC-right atrium junction) IVC enlargement is observed in the presence of normal right atrial pressure [6]. Furthermore, the accuracy of IVC assessment depends on patient's body shape and also on examiner's technique [7-8]. In clinical practice, isolated IVC dilation may be noticed among patients without any other echocardiographic abnormalities [9].

There are several reports assessing the impact of the menstrual cycle on echocardiographic parameters. Changes in female sex hormones level may affect left ventricular diastolic function without significant influence on the right and left atrial volumes, left ventricular volumes and ejection fraction [10]. Our data show that in the population of young, healthy women, IVC diameter is dependent on the phase of menstrual cycle. First days of menstruation are associated with the larger dimension of IVC, which corresponds with the lowest level of vasodilatory sex hormones (estrogen, progesterone) during that phase [11-12]. We did not

\section{References}

Table 1. Studied group characteristics

\begin{tabular}{lll}
\hline Age (years) & $23.7 \pm 4.2$ & \\
\hline BMI $\left(\mathbf{k g} / \mathbf{m}^{2}\right)$ & $20.97 \pm 2.4$ & \\
\hline & PHASE M & PHASE L \\
\hline $\begin{array}{l}\text { d-IVC (cm) } \\
\text { Respiratory }\end{array}$ & $1.98 \pm 0.25$ & $1.86 \pm 0.3$ \\
\hline \begin{tabular}{l} 
collapsibility (\%) \\
\hline
\end{tabular} & & $72.9 \pm 25.3$ \\
\hline
\end{tabular}

observe similar correlation with IVC respiratory variation and cycle phase. Those discrepancies may arise from the less repeatability of IVC respiratory collapsibility measurements. Accuracy of the IVC diameter assessment during inspiration can be affected by respiratory motion in the position of the IVC [13]. Interestingly, over one third of the studied group had d-IVC exceeding reference values at least in one examination as an isolated abnormality in echocardiography. Therefore, it seems to be justified to extend the diagnostics with a question about menstrual cycle phase.

\section{Conclusions}

Pilot results of our study shows that in the population of young women diameter of inferior vena cava exceeding reference values can be observed frequently. IVC dimension changes correspond with the menstrual cycle phases. However, it does not affect the IVC collapsibility during inspiration. The significance of this observation is not clear and requires further studies on larger groups.

1. Ciozda W, Kedan I, Kehl DW, Zimmer R, Khandwalla R, Kimchi A. The efficacy of sonographic measurement of inferior vena cava diameter as an estimate of central venous pressure. Cardiovasc Ultrasound. 2015;14(1):33.

2. Rudski LG, Lai WW, Afilalo J, Hua L, Handschumacher MD, Chandrasekaran K, et al. Guidelines for the Echocardiographic Assessment of the Right Heart in Adults: A Report from the American Society of Echocardiography: Endorsed by the European Association of Echocardiography, a registered branch of the European Society of Cardiology, and the Canadian Society of Echocardiography. J Am Soc Echocardiogr. 2010 Jul 1;23(7):685-713.

3. Tolbert T, Oparil S. Cardiovascular effects of estrogen. Am J Hypertens. 2001;14(S3):186S-193S.

4. dos Santos RL, da Silva FB, Ribeiro RF, Stefanon I. Sex hormones in the cardiovascular system. Horm Mol Biol Clin Investig. 2014;18(2):89-103.

5. Reed BG, Carr BR. The normal menstrual cycle and the control of ovulation [Internet]. Endotext [Internet]. MDText. com, Inc.; 2015. Available from: https://www.ncbi.nlm.nih.gov/books/NBK279054/?report=reader

6. Beigel R, Cercek B, Luo H, Siegel RJ. Noninvasive evaluation of right atrial pressure. J Am Soc Echocardiogr. 2013;26(9):1033-42.

7. Brennan JM, Blair JE, Goonewardena S, Ronan A, Shah D, Vasaiwala S, et al. Reappraisal of the use of inferior vena cava for estimating right atrial pressure. J Am Soc Echocardiogr. 2007;20(7):857-61.

8. Mookadam F, Warsame TA, Yang HS, Emani UR, Appleton CP, Raslan SF. Effect of positional changes on inferior vena cava size. Eur J Echocardiogr. 2011;12(4):322-5.

9. Kim J-J, Cho K-I, Kang J-H, Goo J-J, Kim K-N, Lee J-Y, et al. Isolated dilatation of the inferior vena cava. Korean J Intern Med. 2014;29(2):241-5.

10. Fuenmayor AJ, Ramírez L, Fuenmayor AM. Left ventricular function and autonomic nervous system balance during two different stages of the menstrual cycle. Int J Cardiol. 2000;72(3):243-6.

11. White RE. Estrogen and vascular function. Vascul Pharmacol. 2002;38(2):73-80.

12. Barbagallo M, Dominguez LJ, Licata G, Shan J, Bing L, Karpinski E, et al. Vascular effects of progesterone: role of cellular calcium regulation. Hypertension. 2001;37(1):142-7.

13. Catherine O, Otto M. Textbook of clinical echocardiography. Philadelphia: WB Saunders; 2018. 